\title{
Unlocking the Potential of Flexible Energy Resources to Help Balance the Power Grid
}

\section{Journal Article}

\section{Author(s):}

Mueller, Fabian L.; Woerner, Stefan; Lygeros, John (iD

Publication date:

2019-09

Permanent link:

https://doi.org/10.3929/ethz-b-000363622

Rights / license:

In Copyright - Non-Commercial Use Permitted

Originally published in:

IEEE Transactions on Smart Grid 10(5), https://doi.org/10.1109/TSG.2018.2878998 


\title{
Unlocking the Potential of Flexible Energy Resources to Help Balance the Power Grid
}

\author{
Fabian L. Müller, Stefan Woerner, and John Lygeros, Fellow, IEEE
}

\begin{abstract}
Flexible energy resources can help balance the power grid by providing ancillary services. However, the balancing potential of most types of resources is restricted by physical constraints such as the size of their energy buffer, power ramp-rate limits, or control delays. Using the example of Secondary Frequency Regulation, this paper shows how the flexibility of energy resources can be exploited more efficiently by considering multiple resources with complementary physical properties and controlling them in a coordinated way. To this end, optimal adjustable control policies are computed based on robust optimization. Our problem formulation takes into account power ramp-rate limits explicitly, and accurately models the different timescales and lead times of the energy and reserve markets. Simulations demonstrate that aggregating select resources can lead to a synergy effect that frees up additional regulation capacity in the range of $18-1190 \%$ compared with the amount of reserves the systems could provide individually. This synergy effect can unlock the regulation potential of energy resources with control delays or restrictive energy or ramp-rate constraints.
\end{abstract}

Index Terms-Aggregation, frequency regulation, robust optimization

\section{NOMENCLATURE}

$\begin{array}{ll}\text { Acronyms } \\ \text { AG } & \text { Aggregator } \\ \text { LP } & \text { Linear Program } \\ \text { PEV } & \text { Plug-in electric vehicle } \\ \text { SFR } & \text { Secondary Frequency Regulation } \\ \text { SO } & \text { System operator }\end{array}$

\section{Parameters}

$a^{(B)}, b^{(B)}, c^{(B)}$ System parameters of the battery

$a^{(F)}, b^{(F)}, c^{(F)}$ System parameters of the freezer

$c^{\mathrm{DA}}, c^{\mathrm{ID}} \quad$ Day-ahead, intra-day energy prices

$c^{\mathrm{up}}, c^{\mathrm{dn}} \quad \mathrm{Up}$-, down-regulation energy prices

$c^{\text {RES }} \quad$ SFR capacity reservation prices

$e^{\mathrm{DA}}, e^{\mathrm{ID}} \quad$ Day-ahead, intra-day energy schedule

$e^{\mathrm{up}}, e^{\mathrm{dn}}$

$E^{\mathrm{DA}}, E^{\mathrm{ID}}$

Up-, down-regulation energy

$j, J \quad$ System index, total number of systems

$l \quad$ Index of SFR activation time steps

$N^{\mathrm{DA}}, N^{\mathrm{ID}}, N^{\mathrm{S}}$ Number of day-ahead, intra-day, system time steps

$\underline{p}, \bar{p} \quad$ Lower, upper bound on power

F. L. Müller is with the Automatic Control Laboratory, ETH Zurich, Zurich, Switzerland, and IBM Research-Zurich, Rueschlikon, Switzerland. fmularich.ibm.com

S. Woerner is with IBM Research-Zurich, Rueschlikon, Switzerland. worezurich.ibm. com

J. Lygeros is with the Automatic Control Laboratory, ETH Zurich, Zurich, Switzerland. jlygeros@ethz.ch

$Q^{(j)}, q^{(j)}$
$Q^{\mathrm{DA}}, q^{\mathrm{DA}}$
$Q^{\mathrm{ID}}, q^{\mathrm{ID}}$
$\underline{r}, \bar{r}$
$s$
$t$
$T^{\mathrm{C}}$
$T^{\mathrm{dis}}$
$T^{\mathrm{DA}}$
$T^{\mathrm{H}}$
$T^{\mathrm{ID}}$
$T^{\mathrm{RES}}$
$T^{\mathrm{S}}$
$u$
$w$
$\tilde{w}$
$\underline{x}, \bar{x}$
$x_{0, \min }, x_{0, \max }$
$\gamma_{s}^{(j)}$
$\gamma_{\max }^{(j)}$
$\delta^{(j)}$
$\theta_{\text {in }}, \theta_{\text {out }}$
$\underline{\theta}_{\text {in }}, \bar{\theta}_{\text {in }}$
$\sigma^{\mathrm{SFR}}$

Parameters of power reference of system $j$ Day-ahead trading policy parameters Intra-day trading policy parameters Lower, upper bound on power ramp-rate Index of system time steps Continuous time

Discretization time of SFR activation

Discharge time

Timescale of the day-ahead market

Length of planning horizon

Timescale of the intra-day market

SFR tendering period

Discretization time of system dynamics

Exogenous uncontrollable inputs

Discrete SFR activation on timescale $T^{\mathrm{C}}$

Avg. SFR activation on timescale $T^{\mathrm{S}}$

Lower, upper bound on system state

Min., max. initial system state

SFR capacity of system $j$ in time step $s$

Max. SFR capacity avail. from system $j$

Control delay of system $j$

In-, outdoor air temperature

Lower, upper bound on indoor air temp. SFR synergy factor

\section{Functions}

$C^{E}(\cdot)$
$C^{(j)}(\cdot)$
$\mathbf{E}[\cdot]$
$p^{\mathrm{DA}}(\tilde{w}), p^{\mathrm{ID}}(\tilde{w})$
$p_{\text {ref }}(\tilde{w})$
$p_{\text {ref }}(t)$
$p_{\text {tgt }}(t, w(t))$
$R^{\mathrm{REG}}(\cdot)$
$R^{\mathrm{RES}}(\cdot)$
$V(\cdot)$
$w(t)$

Energy procurement costs

Flexibility cost of system $j$

Expectation

Day-ahead, intra-day power trades

Breakpoint values of power reference

Reference power trajectory at time $t$

Target power trajectory at time $t$

Regulation energy remuneration

SFR reservation remuneration

Profit made by AG

SFR activation at time $t$

Sets

$\mathbb{N}^{s} \quad$ Set of integers $1,2, \ldots, s$

$\mathbb{T}^{\mathrm{DA}}, \mathbb{T}^{\mathrm{ID}}$ Day-ahead, intra-day market time interval

$\mathbb{P}\left(\Phi^{(j)}\right) \quad$ Set of feasible power trajectories of system $j$

$\mathbb{W} \quad$ Set of possible SFR activations

$\zeta^{(j)} \quad$ Decision variables of system $j$

$\Phi^{(j)} \quad$ Parameters of system $j$

$\Psi \quad$ Set of uncertain param. in the bidding problem

$\Omega \quad$ Set of decision variables in bidding problem 


\section{INTRODUCTION}

$\mathbf{T}$ O guarantee the safe and reliable operation of the electricity grid, the system operator (SO) depends on different types of ancillary services. In particular, the Secondary Frequency Regulation (SFR) service is invoked by the SO to reestablish the nominal grid frequency and to compensate for mismatches of energy schedules on the timescale of seconds to minutes [1], [2]. SFR has traditionally been provided by precisely controllable large-scale generating units, such as fossil-fired steam and combutions turbines, as well as nuclear and hydro power plants. With growing shares of renewable intermittent energy resources, such as wind and solar, the need for rapid and accurate SFR is increasing. To support the traditional providers of SFR, various types of electric energy resources have been identified whose flexibility in producing or consuming electricity can be used for SFR. In particular, the balancing potential of heating and cooling systems [3]-[6], plug-in electric vehicles (PEVs) [7], [8], and systems behaving like batteries [9] has been studied extensively.

The potential of individual systems to provide SFR can be severely limited by their physical constraints. For instance, PEVs batteries, domestic heating and cooling systems, capacitors, and flywheels are mainly limited by the size of their energy buffers [6], [10], while steam turbines and industrial cooling systems must adhere to restrictive power ramp-rate limits [11]. Moreover, operational constraints, such as the trips of PEVs, run-time constraints, and control delays, can have significant impact on the ability of a system to provide SFR. In addition to the physical and operational constraints, systems offering SFR services must satisfy the terms and conditions dictated by the energy and reserve markets, such as market timescales and lead times, as well as particular requirements on bids, such as time-invariant and symmetric reserve bids [1]. Approaches to remedy these issues fall into two main groups. The approaches of the first group propose new SFR products that are better suited for the kind of systems listed above, for example by limiting the energy content [12]-[14] or the frequency bandwith of SFR products [5], [15], [16], or by reducing the time period over which SFR capacity must be available [3]. The second group of approaches relies on aggregating the flexibility of multiple systems and controlling them in a coordinated way. This has proven useful to alleviate the difficulties related to the duration and symmetry requirements of certain SFR markets [3], [17]. The same aggregation techniques can be used to couple energy storage systems with energy markets, allowing the former to adjust their energy schedules via the markets and, thereby, to exploit their flexibility more efficiently [4], [10].

In a market setting, each group of systems is managed by an aggregator (AG) who acts as its representative and trades energy and ancillary services on respective markets with the goal of making an economic profit [4], [18]. We refer to the problem of making optimal decisions on energy and reserve markets as the energy and reserve bidding problem. The problem has been approached by means of Model Predictive Control [19], stochastic optimization [13], [20], and robust optimization [3], [14], [21], [22], among others. The robust formulations rely on affinely adjustable robust control policies, which were first applied to the regulation power context in [23], [24], building on the initial work in [25].

In contrast to power and energy constraints, the power ramp-rate constraints are commonly neglected in the literature when solving the energy and reserve bidding problem. This is a major shortcoming because the accurate provision of SFR services can be compromised by insufficient ramping capabilities. The crucial importance of incorporating ramping constraints has already been recognized in the context of grid balancing [15] and unit commitment [26], [27], and has even lead to the adoption of new performance-based schemes for the remuneration of SFR provision by several SOs [28].

In our formulation of the bidding problem, we adopt the robust formulations of [3], [22], [23], [29], and make two main contributions. First, building on the mathematical foundations developed in our previous work (for single systems) [30], we formulate the energy and reserve bidding problem for an aggregation of multiple systems. In contrast to existing approaches, such as [3], [22], [23], [29], our approach allows us to properly model and aggregate the flexibility of systems that are subject to power ramp-rate and control delays, and to consider all the different timescales of the energy and reserve markets. Second, we demonstrate that aggregating multiple systems with complementary physical properties makes it possible to unlock significant amounts of SFR capacity. Simulations show that aggregating fast-responding but energyconstrained systems, such as batteries or capacitors, and slowly-responding systems with large energy buffers, such as industrial heating or cooling systems or steam turbines, yield an increase in the available SFR capacity in the range of 18$1190 \%$ depending on system parameters.

The energy and reserve bidding problem is introduced in Section II. Section III discusses the adjustable control setup used. The electric flexibility of individual system subject to power, power ramp-rate, and energy constraints is provided in Section IV. It is used in Section V to formulate the aggregate bidding problem as a robust optimization problem. In Sections VI and VII, we present the results from aggregating the flexibility of systems with complementary physical properties. Conclusion are provided in Section VIII.

\section{Problem Description}

We consider an AG that controls the electricity production or consumption of a group of flexible energy resources, referred to as a balance group, over a planning horizon of duration $T^{\mathrm{H}}$. The AG can exploit the collective flexibility of the balance group when trading on energy and reserve markets.

\section{A. Energy markets}

To satisfy the energy requirements of the systems in its balance group, the AG can trade electric energy prior to delivery. On the day-ahead market, energy is traded once a day for every time interval of duration $T^{\mathrm{DA}}$ of the next day (usually $T^{\mathrm{DA}}=1 \mathrm{~h}$ ). We denote by $e^{\mathrm{DA}} \in \mathbb{R}^{N^{\mathrm{DA}}}$, $N^{\mathrm{DA}}:=T^{\mathrm{H}} / T^{\mathrm{DA}}$, the final energy schedule resulting from all the day-ahead market trades executed during the planning 
horizon $T^{\mathrm{H}}$. To make adjustments to the day-ahead energy schedule during a particular day, the intra-day market allows trading energy continuously for time intervals of durations $T^{\mathrm{ID}}$ (usually $T^{\mathrm{ID}}=5-15 \mathrm{~min}$ ). All intra-day energy trades are summarized by $e^{\mathrm{ID}} \in \mathbb{R}^{N^{\mathrm{ID}}}, N^{\mathrm{ID}}:=T^{\mathrm{H}} / T^{\mathrm{ID}}$. The total energy procurement costs are $C^{E}:=c^{\mathrm{DA}^{\top}} e^{\mathrm{DA}}+c^{\mathrm{ID}^{\top}} e^{\mathrm{ID}}$, where $c^{\mathrm{DA}} \in \mathbb{R}^{N^{\mathrm{DA}}}$ and $c^{\mathrm{ID}} \in \mathbb{R}^{N^{\mathrm{ID}}}$ denote the energy prices, which are unknown at the time of bidding.

The AG is assumed to be balance-responsible, i.e., it must ensure that the energy production/consumption of its balance group complies with the net energy schedule resulting from the markets. The AG is held accountable for any mismatches between the actual and planned energy schedule.

\section{B. Reserve markets}

The SO constantly observes the state of the power grid and, in case of imbalances, takes measures to reestablish the nominal operating state. To be able to compensate fluctuating generation and consumption and other unexpected disturbances, it procures ancillary services on reserve markets ahead of time [2]. We focus on SFR [1], [2] and assume that symmetric reserve capacities $\gamma_{s} \in \mathbb{R}_{0}^{+}$are traded for all time intervals of duration $T^{\mathrm{S}}$ in the SFR tendering period $T^{\mathrm{RES}}$. The approach can be generalized to asymmetric reserves [14]. For simplicity, we will use $T^{\mathrm{RES}}=T^{\mathrm{H}}$ and, thus, $s=1, \ldots, N^{\mathrm{S}}:=T^{\mathrm{H}} / T^{\mathrm{S}}$. If the capacity bid $\gamma_{s}$ of an AG is accepted, the SO has the right, but not the obligation, to ask the AG to deviate from the planned power reference by $\gamma_{s} w(t)$ units of power at any time during the corresponding time interval $\left[(s-1) T^{\mathrm{S}}, s T^{\mathrm{S}}\right)$. The SFR activation signal $w(t)$ is limited to the interval $\mathbb{W}:=[-1,1]$ and is unknown ahead of time. In return for keeping the reserve capacity $\gamma:=\left[\gamma_{1}, \ldots, \gamma_{N^{s}}\right]^{\top}$ available over the horizon $T^{\mathrm{H}}$, the AG receives the capacity reservation payment $R^{\mathrm{RES}}:=c^{\mathrm{RES}^{\top}} \gamma$, where $c^{\mathrm{RES}}$ are the reserve capacity prices, which are unknown at the time of bidding [1]. Power deviations due to SFR activation can result in energy deviations from the planned energy schedule of an AG. This socalled up- and down-regulation energy is usually measured over each time interval $T^{\mathrm{ID}}$ separately for positive and negative activation, and is denoted by $e^{\text {up }}$ and $e^{\mathrm{dn}}$, respectively [1]. The AG is remunerated for providing SFR based on the amount of up- and down-regulation energy delivered, i.e., the remuneration is $R^{\mathrm{REG}}:=c^{\mathrm{up}^{\top}} e^{\mathrm{up}}-c^{\mathrm{dn}} e^{\mathrm{dn}}$, where $c^{\mathrm{up}}, c^{\mathrm{dn}} \in \mathbb{R}^{N^{\mathrm{ID}}}$ are the corresponding regulation energy prices [1]. However, recent regulatory changes in the USA require all SOs to base their SFR compensation payment not only on the amount of regulation power reserved and regulation energy delivered, but also on the achieved accuracy of up- and down-regulation [28], [31]. This motivates us to impose strict requirements on SFR performance, see III-A.

\section{The bidding problem}

The energy and reserve bidding problem of the AG is to decide how to trade on the energy markets and how much reserve capacity to offer on the SFR market so as to maximize its profit. The profit made by the aggregator is given by

$$
V(\Omega, \Psi):=R^{\mathrm{RES}}(\Omega)+R^{\mathrm{REG}}(\Omega, \Psi)-C^{E}(\Omega, \Psi)
$$

and is a function of the AG's decision variables $\Omega:=\left\{\gamma, e^{\mathrm{DA}}, e^{\mathrm{ID}}\right\}$, and of all the uncertain parameters $\Psi:=\left\{c^{\mathrm{DA}}, c^{\mathrm{ID}}, c^{\mathrm{RES}}, c^{\mathrm{up}}, c^{\mathrm{dn}}, e^{\mathrm{up}}, e^{\mathrm{dn}}\right\}$, which are unknown at the time of bidding. However, an empirical estimate of the probability density of $\Psi$ is assumed to be known. We consider the AG to be a price taker in both the energy and the reserve markets and consider the expected profit $\mathbf{E}_{\Psi}[V(\Omega, \Psi)]$. The problem of finding optimal bids in terms of price-quantity pairs is out of the scope of this work. It has been studied in [32], [33], among others. A particular choice of $\Omega$ together with a realization of the SFR activation signal $w$ results in a unique power trajectory $p(\Omega, w)$ that the balance group of the AG is committed to implement. The AG must choose $\Omega$ subject to the constraints that the energy needs of the systems in the balance group are satisfied and the offered SFR capacity is available over the entire planning horizon for any realization of the uncertain SFR activation $w \in \mathbb{W}$. The energy and reserve bidding problem can be written as

$$
\begin{array}{cl}
\max _{\Omega} & \mathbf{E}_{\Psi}[V(\Omega, \Psi)] \\
\text { s.t. } & p(\Omega, w) \text { feasible } \forall w \in \mathbb{W} .
\end{array}
$$

The more reserve capacity $\gamma$ the AG offers, the more restrictive become the constraints $(2 \mathrm{~b})$ with regard to possible energy trades $e^{\mathrm{DA}}, e^{\mathrm{ID}}$. Consequently, offering SFR reserves and trading energy involves a trade-off between the reserve reward $R^{\mathrm{RES}}$, the potential remuneration of regulation energy $R^{\mathrm{REG}}$, and the energy procurement costs $C^{E}$.

Problem (2) is a robust optimization problem because the constraints (2b) must hold for any admissible realization of the SFR activation signal $w$, see [34]. In Section III, we provide the details of the mapping $p(\Omega, w)$ based on which we derive a robust reformulation of the constraint (2b) in Section IV. This allows us to rewrite the robust bidding problem (2) as a standard Linear Program (LP) in Section V.

\section{Different timescales}

The bidding problem takes place on different timescales. Trading decisions are made on the timescales $T^{\mathrm{RES}}, T^{\mathrm{DA}}$, and $T^{\mathrm{ID}}$ corresponding to the reserve market, and the dayahead and the intra-day energy market, respectively. Online activation of SFR occurs at the faster timescale $T^{\mathrm{C}}$. The dynamics of individual systems are time-discretized on an intermediate timescale $T^{\mathrm{S}}$ commonly chosen in the range of 5-15 min in the case of SFR. In general, it holds that $T^{\mathrm{H}} \geq T^{\mathrm{RES}} \geq T^{\mathrm{DA}} \geq T^{\mathrm{ID}} \geq T^{\mathrm{S}} \gg T^{\mathrm{C}}$. For simplicity, we assume that longer time horizons are integer multiples of shorter ones, and for $* \in\{$ RES, DA, ID, S, C $\}$ define $N^{*}:=T^{\mathrm{H}} / T^{*}$, and $\mathbb{T}^{*}(s)$ the continuous time interval $\left[(s-1) T^{*}, s T^{*}\right)$. Finally, let $\mathbb{N}^{s}:=\{1,2, \ldots, s\}$ for $s \in \mathbb{N}^{+}$. Different timescales are used in different market regions. All timescales $T^{*}$ are used as parameters in our approach making it versatile and applicable to the various market settings. Here we consider the Swiss market setting and use $T^{\mathrm{H}}=T^{\mathrm{RES}}=1$ week, $T^{\mathrm{DA}}=1$ $\mathrm{h}, T^{\mathrm{ID}}=15 \mathrm{~min}, T^{\mathrm{S}}=5 \mathrm{~min}$, and $T^{\mathrm{C}}=1 \mathrm{~s}$. 


\section{Adjustable Power Reference}

\section{A. Power reference and power target}

Every system $j \in \mathbb{N}^{J}$ in the balance group implements a continuous-time piece-wise affine and continuous power reference which is fully defined by its breakpoints $p_{\text {ref }}^{(j)}:=\left[p_{\text {ref }, 0}^{(j)}, \ldots, p_{\text {ref }, N^{\mathrm{s}}}^{(j)}\right]^{\top}$, according to

$$
p_{\text {ref }}^{(j)}(t):=p_{\text {ref }, s-1}^{(j)}+\left(p_{\text {ref }, s}^{(j)}-p_{\text {ref }, s-1}^{(j)}\right)\left(t-(s-1) T^{\mathrm{S}}\right) / T^{\mathrm{S}},
$$

for $t \in \mathbb{T}^{\mathrm{S}}(s), \quad s \in \mathbb{N}^{\mathrm{S}}$. On the aggregate level, the balance group thus implements the reference $p_{\text {ref }}^{(a g g)}(t):=p_{\text {ref }}^{(1)}(t)+\cdots+p_{\text {ref }}^{(J)}(t)$.

The SFR service is activated by the SO by sending out an activation signal to all systems that offer SFR for the corresponding time interval. The activation signal $w:=\left[w_{1}, \ldots, w_{N^{c}}\right]^{\top}$ is a discrete-time signal with $w_{l} \in \mathbb{W}$, $l \in \mathbb{N}^{\mathrm{C}}$. The signal is computed by the SO and broadcast sequentially on the timescale $T^{\mathrm{C}}$ (e.g. , $T^{\mathrm{C}}=1-5 \mathrm{~s}$ in Continental Europe [2], $T^{\mathrm{C}}=1 \mathrm{~s}$ in Switzerland [13], and $T^{\mathrm{C}}=4-6 \mathrm{~s}$ in the USA [28]) such that $w_{l}$ becomes available only at time $(l-1) T^{\mathrm{C}}$. We interpret the discrete signal $w$ as the continuous-time piece-wise affine and continuous activation signal

$$
w(t):=w_{l-1}+\left(w_{l}-w_{l-1}\right)\left(t-(l-1) T^{\mathrm{C}}\right) / T^{\mathrm{C}},
$$

where $t \in \mathbb{T}^{C}(l), l \in \mathbb{N}^{C}$ [35]. We assume that an AG offering the SFR capacity $\gamma^{(\text {agg })} \in \mathbb{R}^{N^{S}}$ is responsible for its balance group to continuously track the target power level

$$
p_{\mathrm{tgt}}^{(a g g)}(t, w):=p_{\mathrm{ref}}^{(a g g)}(t)+\gamma_{s}^{(a g g)} w(t),
$$

with $t \in\left[0, T^{\mathrm{H}}\right]$ and $s \in \mathbb{N}^{\mathrm{S}}: t \in \mathbb{T}^{\mathrm{S}}(s)$. This assumption is more restrictive than current European regulations that only require tracking $w(t)$ within a certain tolerance [36]. However, the accurate provision of SFR is not only desirable from a gridbalancing perspective, but it is already considered in the SFR compensation payment by SOs in the USA, see Section II-B. Inaccurate provision of SFR thus reduces the profit made by the $A G$ and can even lead to disqualification.

The SFR delivered by an AG is the result of a collective effort of all the systems in its balance group, i.e., $\gamma^{(a g g)}:=\gamma^{(1)}+\cdots+\gamma^{(J)}$. Consequently, each system is required to follow its own target power trajectory

$$
p_{\text {tgt }}^{(j)}(t, w):=p_{\text {ref }}^{(j)}(t)+\gamma_{s}^{(j)} w(t) .
$$

Compared with $p_{\text {ref }}^{(j)}(t)$, which is piece-wise affine on the timescale $T^{\mathrm{S}}$, the activation $w(t)$ can vary at the higher rate $T^{\mathrm{C}}$. Thus, accurate tracking of (5) puts higher requirements on the ramping capabilities and control delays of a system.

\section{B. Adjustable power reference}

To exploit the flexibility of energy resources more efficiently, it has been proposed to adjust the reference power schedules (3) in response to past activation, see [14], [22][24], among others. Let $\tilde{w} \in \mathbb{W}^{N^{\mathrm{S}}}$ denote the activation signal $w(t)$ averaged over time intervals of duration $T^{\mathrm{S}}$, i.e.,

$$
\tilde{w}_{s}:=\frac{1}{T^{\mathrm{S}}} \int_{(s-1) T^{\mathrm{S}}}^{s T^{\mathrm{S}}} w(t) d t, s \in \mathbb{N}^{\mathrm{S}} .
$$

Here we consider power references (3) whose breakpoints $p_{\text {ref }}^{(j)}$ are affinely adjustable based on past SFR activation, i.e.,

$$
p_{\text {ref }}^{(j)}(\tilde{w})=Q^{(j)} \tilde{w}+q^{(j)}, j \in \mathbb{N}^{J},
$$

with parameters $Q^{(j)} \in \mathbb{R}^{\left(N^{\mathrm{S}}+1\right) \times N^{\mathrm{S}}}$ and $q^{(j)} \in \mathbb{R}^{N^{\mathrm{S}}+1}$. Equation (7) is a control policy describing the power reference a system should follow depending on the SFR activation $\tilde{w}$. To be applicable in practice, the policy (7) must be causal, i.e., it must consider the fact that the value of $\tilde{w}_{s}$ becomes known only at time $s T^{\mathrm{S}}$ and may be used exclusively to adjust future breakpoint values $p_{\text {ref }, s+1}^{(j)}, \ldots, p_{\text {ref, } N^{\mathrm{s}}}^{(j)}$. Thus, the first two breakpoints, $p_{\text {ref }, 0}$ and $p_{\text {ref }, 1}$, must be set ahead of time without any knowledge about $\tilde{w}$. That is, $p_{\text {ref, } 0}=q_{1}^{(j)}$ and $p_{\text {ref }, 1}=q_{2}^{(j)}$. Consequently, the first two rows of the matrix $Q^{(j)}$ must be all zeros. At time $T^{\mathrm{S}}$, the first measurement $\tilde{w}_{1}$ is available and can be used to compute $p_{\text {ref, } 2}^{(j)}$. In general, the conditions for the policy (7) to be causal are

$$
Q_{m, n}^{(j)}=0 \quad \forall m \in \mathbb{N}^{\mathrm{S}+1}, n \in \mathbb{N}^{\mathrm{S}}: m \leq n+1,
$$

where $Q_{m, n}^{(j)}$ denotes the $m^{\text {th }}$ element in the $n^{\text {th }}$ column of the matrix $Q^{(j)}$.

\section{Energy balance}

To comply with the energy contracts concluded on the dayahead and intra-day markets, the balance-responsible AG must ensure that its balance group follows the net power references $p^{\mathrm{DA}}$ and $p^{\mathrm{ID}}$ resulting from the day-ahead and intra-day energy markets, respectively, i.e.

$$
p_{\text {ref }}^{(a g g)}(\tilde{w})=p^{\mathrm{DA}}+p^{\mathrm{ID}} \forall \tilde{w} \in \mathbb{W}^{N^{\mathrm{S}}} .
$$

Given our choice of affine policies (7), the balance equation (9) suggests that the day-ahead and intra-day trading decisions be affine policies of $\tilde{w}$ also, i.e.

$$
\begin{aligned}
p^{\mathrm{DA}}(\tilde{w}) & =Q^{\mathrm{DA}} \tilde{w}+q^{\mathrm{DA}}, \\
p^{\mathrm{ID}}(\tilde{w}) & =Q^{\mathrm{ID}} \tilde{w}+q^{\mathrm{ID}} .
\end{aligned}
$$

In this case, the balance equation (9) is satisfied if and only if

$$
\begin{aligned}
Q^{(a g g)} & :=\sum_{j \in \mathbb{J}} Q^{(j)}=Q^{\mathrm{DA}}+Q^{\mathrm{ID}}, \text { and } \\
q^{(a g g)} & :=\sum_{j \in \mathbb{J}} q^{(j)}=q^{\mathrm{DA}}+q^{\mathrm{ID}},
\end{aligned}
$$

with parameters $\quad Q^{\mathrm{DA}}, Q^{\mathrm{ID}} \in \mathbb{R}^{\left(N^{\mathrm{S}}+1\right) \times N^{\mathrm{S}}} \quad$ and $q^{\mathrm{DA}}, q^{\mathrm{ID}} \in \mathbb{R}^{N^{\mathrm{S}}+1}$. The power policies (10) correspond to the energy trading policies

$$
\begin{aligned}
e^{\mathrm{DA}}(\tilde{w}) & =E^{\mathrm{DA}}\left(Q^{\mathrm{DA}} \tilde{w}+q^{\mathrm{DA}}\right), \\
e^{\mathrm{ID}}(\tilde{w}) & =E^{\mathrm{ID}}\left(Q^{\mathrm{ID}} \tilde{w}+q^{\mathrm{ID}}\right),
\end{aligned}
$$

where $E^{\mathrm{DA}} \in \mathbb{R}^{N^{\mathrm{DA}} \times\left(N^{\mathrm{S}}+1\right)}$ and $E^{\mathrm{ID}} \in \mathbb{R}^{N^{\mathrm{ID}} \times\left(N^{\mathrm{S}}+1\right)}$ map power to energy. The AG can use the policies (12) to decide on the amount of energy to trade on the corresponding markets based on past SFR activation data. To be applicable, the policies must, however, be causal and take into account the lead times of the different energy markets. These requirements can be incorporated by imposing particular structures onto the matrices $\left(E^{\mathrm{DA}} Q^{\mathrm{DA}}\right)$ and $\left(E^{\mathrm{ID}} Q^{\mathrm{ID}}\right)$. 


\section{DESCRIPTION OF FLEXIBILITY}

The decisions the AG makes on the different energy and reserve markets are restricted by the requirement that the individual target power trajectories (5) must satisfy all the physical constraints of the corresponding system for all realizations of the unknown activation signal. We consider power, power ramp-rate, and state (e.g. energy) constraints. The system index $(j)$ is omitted to simplify the notation.

\section{A. Power constraints}

The power that the system can consume from or feed into the power grid is limited. For all $t \in \mathbb{T}^{\mathrm{S}}(s), s \in \mathbb{N}^{\mathrm{S}}$, we require that

$$
\underline{p}_{s} \leq p_{\text {tgt }}(t, w) \leq \bar{p}_{s}, \forall w \in \mathbb{W}^{N^{c}},
$$

where $\underline{p}, \bar{p} \in \mathbb{R}^{N^{\mathrm{S}}}$ denote the piece-wise constant bounds on power. The constraints above are satisfied if for all $\tilde{w} \in \mathbb{W}^{N^{\mathrm{s}}}$ and $s=0, \ldots, N^{\mathrm{S}}$ it holds that

$$
\begin{aligned}
p_{\text {ref }, s}(\tilde{w})+\gamma_{\max \{1, s\}} & \leq \bar{p}_{\max \{1, s\}}, \\
p_{\text {ref }, s}(\tilde{w})-\gamma_{\max \{1, s\}} & \geq \underline{p}_{\max \{1, s\}}, \\
p_{\text {ref }, s}(\tilde{w})+\gamma_{\min \left\{N^{\mathrm{s}}, s+1\right\}} & \leq \min \left\{\bar{p}_{s}, \bar{p}_{\min \left\{N^{\mathrm{s}}, s+1\right\}}\right\}, \\
p_{\text {ref }, s}(\tilde{w})-\gamma_{\min \left\{N^{\mathrm{s}}, s+1\right\}} & \geq \max \left\{\underline{p}_{s}, \underline{p}_{\min \left\{N^{\mathrm{s}}, s+1\right\}}\right\} .
\end{aligned}
$$

The above set of constraints comprises an infinite number of inequalities due to its dependency on $\tilde{w}$, and, thus, cannot be included in an optimization problem as is. However, following the approach in [25], [34], and exploiting the linearity of (14) in $\tilde{w}$ and the particular structure of $\mathbb{W}$, allows us to derive a set of equivalent constraints. For example, consider the first inequality in (14) and denote by $Q_{s}$ the $s^{\text {th }}$ row of matrix $Q$. By exploiting

$$
p_{\text {ref }, s}(\tilde{w})=Q_{s} \tilde{w}+q_{s} \leq\left\|Q_{s}\right\|_{1}+q_{s}, \forall \tilde{w} \in \mathbb{W}^{N^{\mathrm{c}}},
$$

the first inequality in (14) is equivalent to

$$
\left\|Q_{s}\right\|_{1}+q_{s}+\gamma_{\max \{1, s\}} \leq \bar{p}_{\max \{1, s\}},
$$

which is independent of $\tilde{w}$. The remaining constraints in (14) can be reformulated similarly.

\section{B. Power ramp-rate constraints}

Power ramp-rate limits play an important role, in particular for providing ancillary services with high accuracy. For all $t \in \mathbb{T}^{\mathrm{S}}(s), s \in \mathbb{N}^{\mathrm{S}}$, we require that the rate of change of the target power level be bounded, i.e.,

$$
\underline{r}_{s} \leq \frac{\partial}{\partial t} p_{\text {tgt }}(t, w) \leq \bar{r}_{s}, \forall w \in \mathbb{W}^{N^{\mathrm{c}}},
$$

where $\underline{r}, \bar{r} \in \mathbb{R}^{N^{\mathrm{S}}}$ denote the piece-wise constant power ramprate limits. The above inequalities are satisfied if and only if for all $\tilde{w} \in \mathbb{W}^{N^{\mathrm{S}}}$ it holds for $s=1, \ldots, N^{\mathrm{S}}$ that

$$
\begin{aligned}
& \left(p_{\operatorname{ref}, s}(\tilde{w})-p_{\operatorname{ref}, s-1}(\tilde{w})\right) / T^{\mathrm{S}}+2 \gamma_{s} / T^{\mathrm{C}} \leq \bar{r}_{s}, \\
& \left(p_{\mathrm{ref}, s}(\tilde{w})-p_{\operatorname{ref}, s-1}(\tilde{w})\right) / T^{\mathrm{S}}-2 \gamma_{s} / T^{\mathrm{C}} \geq \underline{r}_{s},
\end{aligned}
$$

and for $s=1, \ldots, N^{\mathrm{S}}-1$ that

$$
\begin{aligned}
& \left(p_{\text {ref }, s}(\tilde{w})-p_{\operatorname{ref}, s-1}(\tilde{w})\right) / T^{\mathrm{S}}+\left(\gamma_{s}+\gamma_{s+1}\right) / T^{\mathrm{C}} \leq \bar{r}_{s}, \\
& \left(p_{\text {ref }, s}(\tilde{w})-p_{\text {ref }, s-1}(\tilde{w})\right) / T^{\mathrm{S}}-\left(\gamma_{s}+\gamma_{s+1}\right) / T^{\mathrm{C}} \geq \underline{r}_{s} .
\end{aligned}
$$

Constraints equivalent to (16) and (17) but independent of $\tilde{w}$ can be derived as shown in Section IV-A.

\section{State constraints}

Many types of flexible energy resources owe their flexibility to an energy buffer with limited capacity. Examples of such systems are pumped hydro-power plants, batteries, heating and cooling systems, capacitors, and flywheels. Here we consider systems whose energy buffer level $x(t) \in \mathbb{R}$ is governed by the linear time-invariant dynamics

$$
\frac{\partial}{\partial t} x(t, w)=a x(t, w)+b u_{s}+c p_{\mathrm{tgt}}(t, w),
$$

with $t \in \mathbb{T}^{\mathrm{S}}(s), s \in \mathbb{N}^{\mathrm{S}}$. Exogenous (uncontrollable) inputs, such as the weather conditions in the case of heating systems, or trips in the case of electric-vehicle batteries, are summarized by $u:=\left[u_{1}, \ldots, u_{N^{s}}\right]^{\top}$ and are assumed to be piece-wise constant and known. Because the bidding problem has to be solved ahead of time, the initial state $x(0)$ is not known precisely, but is known to lie in the interval $\left[x_{0, \min }, x_{0, \max }\right]$. The dynamics (18) are characterized by the parameters $\{a, b, c\}$, where $a \leq 0$ can be interpreted as the self-dissipation rate of the energy buffer, and $b, c$ determine the conversion efficiencies of exogenous inputs and electric energy into buffered energy, respectively. The behavior of an ideal battery, for instance, is modeled by $a=b=0$ and $c=1$.

We consider the piece-wise constant state constraints

$$
\underline{x}_{s} \leq x(t, w) \leq \bar{x}_{s}, \forall w \in \mathbb{W}^{N^{\mathrm{c}}},
$$

for all $t \in \mathbb{T}^{\mathrm{S}}(s), \quad s=\mathbb{N}^{\mathrm{S}}, x(0) \in\left[x_{0, \min }, x_{0, \max }\right]$, where $\underline{x}, \bar{x} \in \mathbb{R}^{N^{S}}$ denote the physical limits of the system state. Constraints (19) can be interpreted as time-varying limits on the "state-of-charge" of the system. The fact that the target power $p_{\text {tgt }}$ is piece-wise affine on the timescale $T^{\mathrm{C}}$ makes the robust reformulation of (19) nontrivial. Due to space limitations, the interested reader is referred to the detailed discussion in [30], where the robust counterparts of (19) are derived and shown to be linear in the trading policy parameters $\left\{Q^{(j)}, q^{(j)}\right\}$ of an individual resources.

\section{Description of flexibility}

The robust counterparts of the power, ramp-rate, and state constraints (13), (15), and (19), respectively, are linear in the decision variables $\zeta^{(j)}:=\left\{Q^{(j)}, q^{(j)}, \gamma^{(j)}\right\}$, see [30]. Thus, they define a convex polytope $\mathbb{P}\left(\Phi^{(j)}\right)$ parameterized by the system constraints and dynamics parameters $\Phi^{(j)}:=$ $\left\{\underline{p}^{(j)}, \bar{p}^{(j)}, \underline{r}^{(j)}, \bar{r}^{(j)}, \underline{x}^{(j)}, \bar{x}^{(j)}, x_{0, \min }^{(j)}, x_{0, \max }^{(j)}, a^{(j)}, b^{(j)}, c^{(j)}\right\}$. The polytope $\mathbb{P}\left(\Phi^{(j)}\right)$ can serve as a concise and convenient description of the flexibility system $j$ offers with regard to trading energy and providing SFR services. 


\section{The Bidding Problem}

\section{A. Formulation of the bidding problem}

Based on our model of the adjustable power references and the reformulation of the robust power, power ramp-rate and state constraints provided above, we can rewrite the robust energy and reserve bidding problem (2) as a standard LP. The problem consists of finding the power reference policies (7), the trading policies (10), and the SFR capacities $\gamma^{(j)}$ that maximize the expected profit while satisfying all the system and market constraints, i.e.,

$$
\begin{array}{ll}
\max _{\Omega} & \mathbf{E}_{\Psi}[V(\Omega, \Psi)] \\
\text { s.t. } & \zeta^{(j)} \in \mathbb{P}\left(\Phi^{(j)}\right), j \in \mathbb{N}^{J}, \\
& \sum_{j \in \mathbb{N}^{J}} Q^{(j)}=Q^{\mathrm{DA}}+Q^{\mathrm{ID}}, \\
& \sum_{j \in \mathbb{N}^{J}} q^{(j)}=q^{\mathrm{DA}}+q^{\mathrm{ID}},
\end{array}
$$$$
\text { Causality of (7) via (8), }
$$

Market constraints,

where $\Omega:=\left\{\zeta^{(1)}, \ldots, \zeta^{(J)}, Q^{\mathrm{DA}}, q^{\mathrm{DA}}, Q^{\mathrm{ID}}, q^{\mathrm{ID}}\right\}$ summarizes all the decision variables. The constraints $(20 \mathrm{~b})$ ensure the feasibility of the target power trajectory (5) with respect to the constraints of the inidividual system $j \in \mathbb{N}^{J}$. The constraints (20c) and (20d) guarantee that the AG complies with all energy market trades. The power reference policies must be causal (20e), and respect all market constraints (20f), such as market lead times or the requirement of time-invariant reserve bids.

The constraints of the bidding problem (20) can easily be augmented. For example, special requirements of energy or reserve markets can make it necessary to add marketspecific constraints. For example, some reserve markets, such as the Swiss SFR market [1], require the reserve bids to be constant over the tendering preiod. This can be considered by adding the auxiliary decision variable $\gamma^{(a g g)}$ and the constraints $\gamma_{s}^{(1)}+\cdots+\gamma_{s}^{(J)}=\gamma^{(a g g)}, s \in \mathbb{N}^{S}$. In addition, regulations in continental Europe require that energy schedules are implemented as piece-wise affine and continuous power trajectories that alternate between linear ramps and periods of constant power [2]. These requirements can be formulated as linear constraints on $Q^{\mathrm{DA}}, q^{\mathrm{DA}}, Q^{\mathrm{ID}}, q^{\mathrm{ID}}$, see [30].

\section{B. Complexity of the bidding problem}

The number of decision variables and constraints of problem (20) is determined by various factors, such as the length of the planning horizon, the system discretization time, the different market timescales, and also the structure of the $Q$-matrices that determine the power reference and trading policies (7) and (10), respectively. For instance, the problem size can be reduced significantly, if the power reference policy (7) considers only the $r \geq 1$ most recent measurements of the SFR activation signal instead of its entire history. That is, in addition to (8) we require that

$$
Q_{m, n}^{(j)}=0 \forall m \in \mathbb{N}^{\mathrm{S}+1}, n \in \mathbb{N}^{\mathrm{S}}: m>n+r+2,
$$

which imposes a band structure onto $Q^{(j)}$. The effects of different policies on the solution of problem (20) and the required solving time have been investigated in [23], [30].

Note that problem (20) is an LP that can be solved by standard optimization software. In the numerical study below we use IBM ILOG $\AA^{1}$ CPLEX $\mathbb{R}^{1}$ to solve the LP (20). For larger instances it may be more efficient to solve the problem with distributed optimization methods such as ADMM. The problem lends itself to the use of such methods, as it involves a decomposable objective, and the linear coupling constraints (20c) and (20d), see [17], [37].

\section{The cost of flexibility}

Exploiting the flexibility of electric energy units can have adverse impacts on the systems and their owners. For example, heating and cooling systems deviating from set point profiles can lead to discomfort, and forcing batteries or gas turbines to track volatile SFR activation signals can cause battery degradation [38], put high thermal stress on turbine components, and entail higher plant operations and maintenance costs [39]. Thus, AGs conclude contracts with individual system owners, defining the specifics of the flexibility offered by the systems, as well well as the details on how the systems are reimbursed for the flexibility provided [18], [40]. For example, the flexibility reimbursement of individual systems could be based on flexibility cost functions $C^{(j)}$ [41] that assign a cost to the family of possible power references (7) and power target trajectories (5) that a system $j$ might be required to follow for a given choice of the decision variables $\left\{Q^{(j)}, q^{(j)}, \gamma^{(j)}\right\}$. The AG can consider the cost of flexibility when solving the energy and reserve bidding problem by augmenting its profit function (1) by the negative flexibility costs $-C^{(j)}, j \in \mathbb{N}^{J}$.

\section{SYNERGY I: BATTERIES AND FREEZER}

Aggregating the flexibility of multiple energy resources has been shown to alleviate some of the challenges faced by individual systems, such as limits on the minimum bid size and the requirement of time-invariant SFR bids [3], [17]. Here, we show that aggregating the flexibility of systems with complementary physical properties can significantly increase the amount of SFR capacity that can be offered by the aggregation. We call this the synergy effect of aggregation. To demonstrate this effect, we consider two flexible systems, an ideal battery (B), and an industrial freezer warehouse (F).

\section{A. Battery model}

The battery is modeled as an ideal energy buffer whose state dynamics are governed by (18) with parameters $a^{(B)}=b^{(B)}=0$ and $c^{(B)}=1$. The ramping capabilities of the battery are assumed to be fast enough so that the power ramprate constraints (15) can be neglected. However, the battery is subjected to the power constraints (13) with symmetric timeinvariant limits $-\underline{p}^{(B)}=\bar{p}^{(B)}$, and state constraints (19) with

\footnotetext{
${ }^{1}$ ILOG and CPLEX are trademarks of International Business Machines Corp., registered in many jurisdictions worldwide. Other product and service names might be trademarks of IBM or other companies.
} 
limits $\underline{x}^{(B)}=0, \bar{x}^{(B)}>0$ and $x_{0, \min }^{(B)}=x_{0, \max }^{(B)}=\bar{x}^{(B)} / 2$. The maximum constant and symmetric SFR capacity the battery can offer over an SFR tendering period $T^{\mathrm{RES}}$ can be computed analytically as

$$
\begin{aligned}
\gamma_{\max }^{(B)}=\min \left\{\left|\bar{p}^{(B)}-\underline{p}^{(B)}\right| / 2\right. & ,\left(\bar{x}^{(B)}-x_{0, \max }^{(B)}\right) / T^{\mathrm{RES}}, \ldots \\
& \left.\left(x_{0, \text { min }}^{(B)}-\underline{x}^{(B)}\right) / T^{\mathrm{RES}}\right\} .
\end{aligned}
$$

The maximum constant and symmetric SFR capacity $\gamma_{\max }^{(B)}$ that an individual battery can provide is determined by the most restrictive of three limits. First, the SFR capacity is limited by the battery's power constraints. Because the battery must be able to provide the same amount of up- and down regulation, $\gamma_{\max }^{(B)} \leq\left|\bar{p}^{(B)}-\underline{p}^{(B)}\right| / 2$ is required. The second limiting factor is the battery's energy capacity. In the extreme case where the full up-regulation $-\gamma_{\max }^{(B)}$ is activated over the entire period $T^{\mathrm{RES}}$, the battery must be able to deliver the corresponding energy to the power grid, i.e., $\gamma_{\max }^{(B)} T^{\mathrm{RES}} \leq x_{0, \min }^{(B)}-\underline{x}^{(B)}$. Similarly, if the full downregulation $\gamma_{\max }^{(B)}$ is activated over the entire period $T^{\mathrm{RES}}$, the battery must be able to absorb the corresponding amount of energy, i.e., $\gamma_{\max }^{(B)} T^{\mathrm{RES}} \leq \bar{x}^{(B)}-x_{0 \text { max }}^{(B)}$. In general, the SFR potential of individual batteries is limited by these capacity constraints because their energy capacity is usually small compared to their power rating. Consider for example the battery of a Tesla Model-S with power rating $\bar{p}^{(B)}=-\underline{p}^{(B)}=17.2 \mathrm{~kW}$, capacity $\bar{x}^{(B)}=100 \mathrm{kWh}$ [42], and initial state $x_{0}^{(B)}=50$ $\mathrm{kWh}$ providing constant and symmetric SFR over a time horizon of $T^{\mathrm{RES}}=24 \mathrm{~h}$. According to (21), the battery can provide $\gamma_{\max }^{(B)}=2.08 \mathrm{~kW}$ of SFR which corresponds to only $12.1 \%$ of the SFR capacity it could provide if the capacity constraints are neglected (namely, $\bar{p}^{(B)}=17.2 \mathrm{~kW}$ ).

\section{B. Freezer model}

The freezer is modeled as a single energy buffer whose state evolves according to (18). It must respect the power, power ramp-rate, and state constraints (13), (15), and (19), respectively. The parameters of the freezer are similar to the ones of the freezer warehouse described in [43] and are given in Table I. The freezer consumes electric energy to keep its indoor air temperature $\theta_{\text {in }}$ within the admissible range $\left[\underline{\theta}_{\text {in }}, \bar{\theta}_{\text {in }}\right]$. The time required to cool the freezer from $\bar{\theta}_{\text {in }}$ down to $\underline{\theta}_{\text {in }}$ at maximum cooling power $\bar{p}^{(F)}$ is assumed to be $6 \mathrm{~h}$. That is, the freezer has an energy storage capacity of $\bar{x}^{(F)}=6 \cdot \bar{p}^{(F)}=1.8 \mathrm{MW}$. Given the constant outdoor air temperature $\theta^{\text {out }}=5^{\circ} \mathrm{C}$, we assume that without cooling the indoor temperature rises from $\underline{\theta}_{\text {in }}$ to $\bar{\theta}_{\text {in }}$ in $T^{\mathrm{dis}}=10 \mathrm{~h}$. The parameters of the freezer state dynamics (18) are

$$
\begin{aligned}
& a^{(F)}=\frac{1}{T^{\mathrm{dis}}} \ln \left(\frac{\bar{\theta}_{\text {in }}-\underline{\theta}_{\text {in }}}{\underline{\theta}_{\text {in }}-\theta_{\text {out }}}\right), \\
& b^{(F)}=a^{(F)} \bar{x}^{(F)} /\left(\underline{\theta}_{\text {in }}-\bar{\theta}_{\text {in }}\right), \\
& c^{(F)}=1 .
\end{aligned}
$$

We assume that the power consumption of the freezer's cooling system can be set to any value in the continuous range $\left[\underline{p}^{(F)}, \bar{p}^{(F)}\right]$. However, power set point changes are not
TABLE I

TIME-INVARIANT FREEZER PARAMETERS

\begin{tabular}{crl}
\hline Symbol & Value & Description \\
\hline$\underline{p}^{(F)}, \bar{p}^{(F)}$ & $0,300 \mathrm{~kW}$ & Min., max. power consumpt. \\
$\underline{r}^{(F)}, \bar{r}^{(F)}$ & $-100,100 \mathrm{~kW} / \mathrm{min}$ & Min., max. power ramp-rate \\
$\underline{x}^{(F)}, \bar{x}^{(F)}$ & $0,1.8 \mathrm{MWh}$ & Min., max. state \\
$\underline{x}_{0, \text { min }}^{(F)}=\bar{x}_{0, \max }^{(F)}$ & $0.9 \mathrm{MWh}$ & Limits on initial state \\
$\underline{\theta}_{\text {in }}, \bar{\theta}_{\text {in }}$ & $-29,-27^{\circ} \mathrm{C}$ & Admissible ind. temp. range \\
$\theta_{\text {out }}$ & $5^{\circ} \mathrm{C}$ & Constant outdoor air temp. \\
$a^{(F)}$ & $-0.0061 \mathrm{~h}^{-1}$ & Self-dissipation rate \\
$b^{(F)}$ & $5.4562 \mathrm{~kW} /{ }^{\circ} \mathrm{C}$ & Heat flux to outside \\
$c^{(F)}$ & 1 & Efficiency of cooling syst. \\
\hline
\end{tabular}

implemented immediately but only after a constant delay $\delta^{(F)}$ which can be determined by measuring the change in the system's power consumption in response to a power set point change. Here we assume that $T^{\mathrm{C}}<\delta^{(F)}<T^{\mathrm{S}}$ which makes it impossible for the freezer to track an SFR target power trajectory (5) because it cannot react on the timescale $T^{\mathrm{C}}$. Consequently, the freezer cannot offer any SFR, i.e. $\gamma_{\max }^{(F)}=0$ $\mathrm{kW}$. A delay can also impact the ability of a system to adjust its power reference based on past SFR activation according to (7). This can be incorporated explicitly in the bidding problem by forcing additional elements of $Q^{(F)}$ to zero:

$$
Q_{m, n}^{(F)}=0 \forall m \in \mathbb{N}^{\mathrm{S}+1}, n \in \mathbb{N}^{\mathrm{S}}: n \geq m-1-\left\lceil\frac{\delta^{(F)}}{T^{\mathrm{S}}}\right\rceil .
$$

\section{Maximizing the aggregate SFR potential}

The results of the bidding problem (20) do not only depend on the market characteristics and technical constraints of the systems, but are also influenced by the bidding strategies of the AGs and the resulting market prices. An economic analysis of the bidding problem can be found in [4]. Here, we focus on the synergy effects of aggregation independent of economic considerations. For that purpose, we compute the maximum time-invariant and symmetric SFR capacity $\gamma_{\max }^{(\text {agg) }} \in \mathbb{R}_{0}^{+}$that can be offered by the aggregation of the freezer and the battery by solving the modified bidding problem:

$$
\begin{aligned}
\gamma_{\max }^{(\mathrm{agg})}:=\max _{\Omega} & \min _{s \in \mathbb{N}^{\mathrm{S}}}\left\{\gamma_{s}^{(B)}+\gamma_{s}^{(F)}\right\} \\
\text { s.t. } & \zeta^{(j)} \in \mathbb{P}\left(\Phi^{(j)}\right), j \in\{B, F\}, \\
& Q^{(B)}+Q^{(F)}=Q^{\mathrm{DA}}+Q^{\mathrm{ID}}, \\
& q^{(B)}+q^{(F)}=q^{\mathrm{DA}}+q^{\mathrm{ID}}, \\
& Q^{\mathrm{DA}}=Q^{\mathrm{ID}}=0, q^{\mathrm{ID}}=0,
\end{aligned}
$$

Causality of (7) via (8),

Control delay constraint (23).

By adding the constraints (24e), we model the case where the aggregate power reference (9) is fixed and cannot be adjusted via trades on energy markets. However, the individual power references $p_{\text {ref }}^{(B)}$ and $p_{\text {ref }}^{(F)}$ are still adjustable according to (7) as long as all adjustments cancel out on the aggregate level. Any market constraints (20f) can be omitted. The control delay of the freezer is taken into account by $(24 \mathrm{~g})$. 
Solving (24) using the Tesla Model-S battery parameters yields the maximum aggregate SFR capacity $\gamma_{\max }^{(a g g)}=\gamma^{(B) *}=9.61 \mathrm{~kW}$, which is $55.9 \%$ of $\bar{p}^{(B)}$ and represents an increase by a factor of 4.6 compared to the amount of SFR the battery can provide individually. The optimal power reference policy parameters are

$$
\begin{aligned}
q_{s}^{(B) *} & =0, q_{s}^{(F) *}=q_{s}^{\mathrm{DA} *}=180.05, s \in \mathbb{N}^{\mathrm{S}}, \\
Q_{m, n}^{(F) *} & =\left\{\begin{array}{l}
7.59 \text { for } m \in \mathbb{N}^{\mathrm{S}+1}, n \in \mathbb{N}^{\mathrm{S}}: m=n+3, \\
0 \text { for } m \in \mathbb{N}^{\mathrm{S}+1}, n \in \mathbb{N}^{\mathrm{S}}: m \neq n+3,
\end{array}\right. \\
Q^{(B) *} & =-Q^{(F) *} .
\end{aligned}
$$

Figure 1 illustrates how the battery and the freezer adjust their power references in response to SFR activation. The first plot shows a real activation signal $w$ which was downsampled from $1 \mathrm{~s}$ to $10 \mathrm{~s}$ resolution over to course of 1 day. The signal is biased toward positive values, in particular during the first half of the planning horizon. The second plot depicts the power reference $p_{\text {ref }}^{(B)}$ of the battery for the case where no SFR is activated (horizontal dashed grey line at $0 \mathrm{~kW}$ ), and for the particular activation shown in the first plot (solid grey line). The solid black line denotes the target power level (4). The power trajectories of the freezer are provided in the third plot. Because $\gamma^{(F) *}=0$, the power reference and power target trajectories of the freezer coincide (solid black line). Note that $p_{\text {ref }}^{(F)}=-p_{\text {ref }}^{(B)}$ as required by the balance constraints (24c) and (24d). The evolution of the battery and freezer states $x^{(B)}$ and $x^{(F)}$ are shown in the two bottom plots. In the case of no activation, the states remain constant at their initial values (dashed grey lines). However, for the activation given, both the battery and the freezer must adjust their power references (solid grey line for $x^{(B)}$ and solid black line for $\left.x^{(F)}\right)$. In this way, the battery is able to keep its actual stateof-charge (solid black line) within its feasible range despite a biased activation signal. The energy content of the SFR activation is absorbed by the freezer, whose reference and target state trajectories coincide (solid black line). By acting as a responsive energy buffer, the freezer contributes to providing SFR on the aggregate level even though it cannot offer any SFR on its own.

\section{Synergy effect of aggregation}

To quantify the benefit of aggregating multiple systems, we define the SFR synergy factor as

$$
\sigma^{\mathrm{SFR}}:=\gamma_{\max }^{(\mathrm{agg})} / \sum_{j \in \mathbb{N}^{J}} \gamma_{\max }^{(j)}-1 \in \mathbb{R}_{0}^{+}
$$

The synergy factor measures the amount of additional SFR capacity that is available from the aggregation relative to the sum of SFR capacities the systems could provide individually. A value $\sigma^{\mathrm{SFR}}=0$ means that aggregating the systems does not yield any benefit with regard to SFR capacity, i.e., the SFR capacity available from the aggregation of systems equals the sum of the SFR capacities the systems can provide individually. In contrast, a value $\sigma^{\mathrm{SFR}}>0$ indicates that aggregation results in a synergy effect and is able to free up SFR capacity that
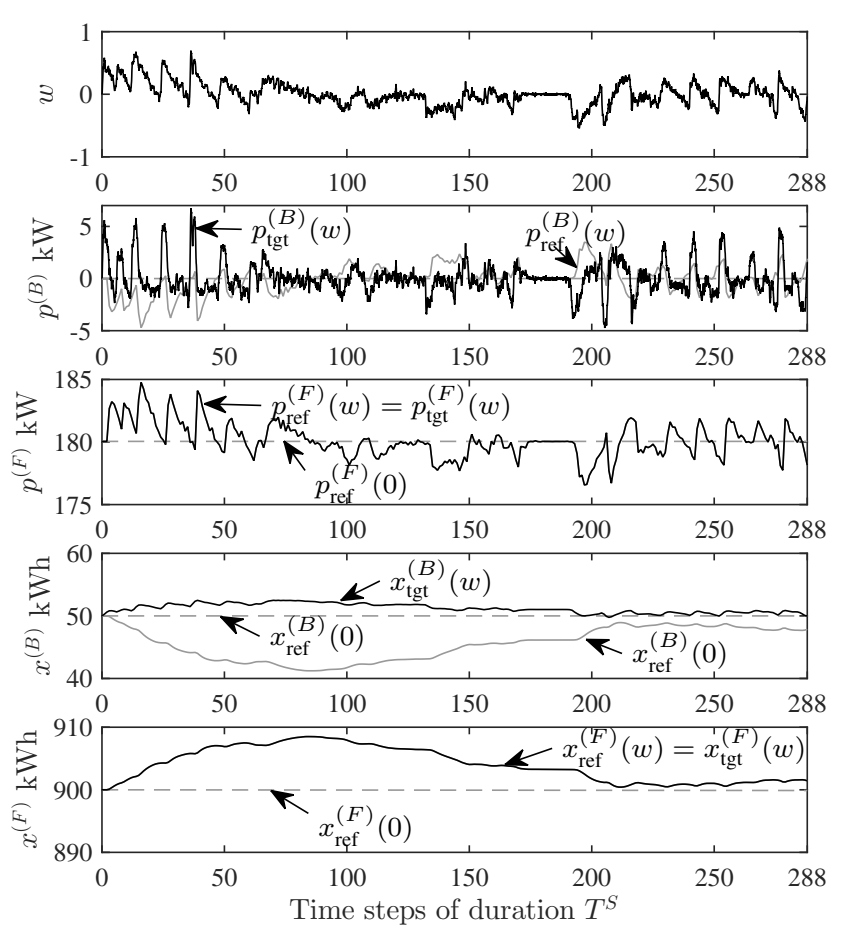

Fig. 1. Power and state trajectories of the battery and the freezer in response to a given activation signal $w$. Reference trajectories in case of no activation $(w=0)$ are drawn as dashed grey lines. The reference trajectories for the given activation are shown as solid gray lines and the target trajectories are drawn as solid black lines. The reference and target trajectories for the freezer coincide because the freezer does not offer any SFR, i.e., $\gamma^{(F) *}=0$.

the systems cannot offer individually. The value $100 \sigma^{\mathrm{SFR}}$ is the increase in available SFR capacity in \%. Figure 2 provides the $\sigma^{\mathrm{SFR}}$-values for the aggregation of a battery and a freezer for different choices of the battery parameters. The circles indicate the battery parameters corresponding to aggregations of $1-5$ Tesla Model-S batteries [42], 1-2 Tesla Powerpack batteries [44], and 2-10 Tesla Powerwall batteries [45]. The battery parameters and the corresponding individual and aggregate maximum SFR capacities and synergy factors are summarized in Table II. Aggregating batteries with a freezer can unlock a significant amount of SFR capacity. Depending on the battery parameters, synergy factors in the range of 3.61-11.90 are achieved. This corresponds to a relative increase in the available SFR capacity by 361-1190\% compared with the case where the systems provide SFR individually.

The synergy factor obtained from coupling batteries with a freezer remains constant for the same power-to-capacity ratio $\bar{p}^{(B)} / \bar{x}^{(B)}$ of the battery. For example, aggregations of 1-5 Model-S batteries and a freezer all result in the same synergy factor $\sigma^{\mathrm{SFR}}=3.61$, see Table II. The same is true for aggregations of 2-10 Powerwall batteries, which all reach synergy factors $\sigma^{\text {SFR }}=11.90$. In these cases, the SFR capacity available on the aggregate level is limited by the battery's power constraints. The capacity constraints are not active because the battery can balance its energy level via the freezer. However, at some point the energy balancing requirement of the battery exceeds the energy capacity of the freezer. This is when the energy capacity constraints of both the battery and the freezer become active simultaneously and limit 


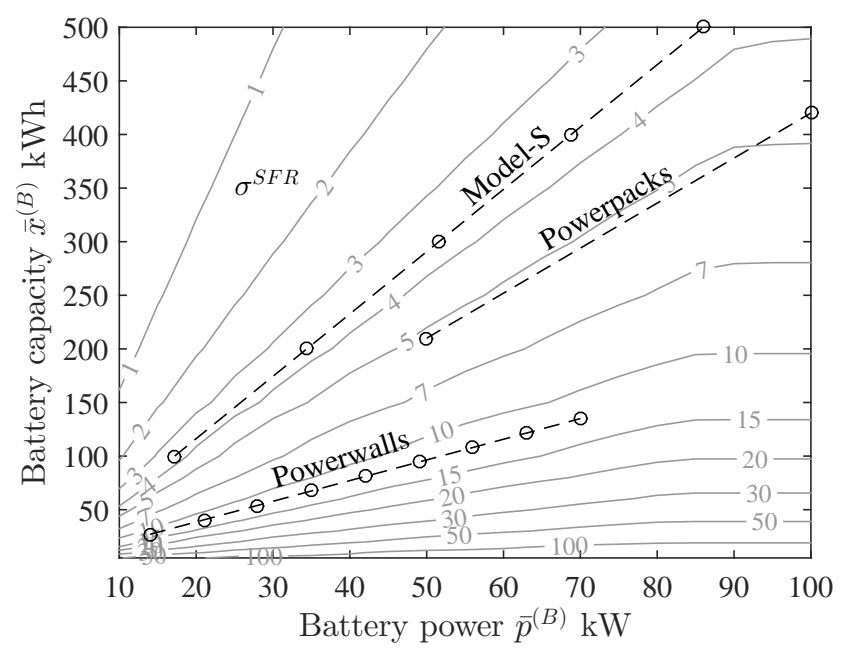

Fig. 2. Values of the synergy factor $\sigma^{\mathrm{SFR}}$ for different choices of the battery parameters $\bar{p}^{(B)}, \bar{x}^{(B)}$ for the aggregation of batteries and a freezer.

TABLE II

BATTERY-FREEZER AGGREGATION

\begin{tabular}{lcccccc}
\hline Battery type & $\#$ & $\begin{array}{c}\bar{p}^{(B)} \\
\mathrm{kW}\end{array}$ & $\begin{array}{c}\bar{x}^{(B)} \\
\mathrm{kWh}\end{array}$ & $\begin{array}{c}\gamma_{\max }^{(\mathrm{B})} \\
\mathrm{kW}\end{array}$ & $\begin{array}{c}\gamma_{\max }^{(\mathrm{agg})} \\
\mathrm{kW}\end{array}$ & $\sigma^{\mathrm{SFR}}$ \\
\hline \hline Model-S & 1 & 17.2 & 100 & 2.08 & 9.61 & 3.61 \\
& 5 & 86 & 500 & 10.42 & 48.04 & 3.61 \\
\hline Powerpack & 1 & 50 & 210 & 4.38 & 27.09 & 5.19 \\
& 2 & 100 & 420 & 8.75 & 49.47 & 4.65 \\
\hline Powerwall & 2 & 14 & 27 & 0.56 & 7.25 & 11.90 \\
& 10 & 70 & 135 & 2.81 & 36.26 & 11.90 \\
\hline
\end{tabular}

the maximum available SFR capacity and the corresponding synergy factor. This effect can be seen using the example of the Powerpacks. Coupling a single Powerpack battery with the freezer yields $\sigma^{\mathrm{SFR}}=5.19$, whereas the aggregation of 2 Powerpacks with a freezer yields only $\sigma^{\mathrm{SFR}}=4.65$. At the points where the energy capacity constraints of the freezer become active, the isolines in Figure 2 turn horizontal and increasing the number of batteries is inefficient because the synergy factor cannot be increased further.

The data shown in Figure 2 can be used by AGs to infer what type of battery should be added to an existing aggregation to increase the synergy factor the most. Consider for example the aggregation of a single Model-S battery and a freezer. The synergy factor can be increased the most by adding more power capacity, i.e., moving horizontally to the right. In the case of two Powerpacks, however, adding more power capacity would not result in a higher synergy factor because the isolines are horizontal. Here, a larger synergy factor can be reached only by augmenting the energy capacity of the freezer.

The results summarized in Figure 2 and Table II show that aggregating systems with complementary physical properties - a fast-responding but energy-constrained battery and a delayed-responding freezer with a comparatively large energy buffer - can unlock significant amounts of SFR capacity. Depending on the battery parameters, a relative increase in the available SFR capacity by $361-1190 \%$ is achieved.

\section{SYNERGY II: BATTERIES AND TURBINE}

Similar synergy effects can be observed when coupling a battery with a system whose ability to offer SFR is restricted by power ramp-rate limits. Consider a steam turbine (ST) with the time-invariant power limits $\underline{p}^{(S T)}=0 \mathrm{MW}$ and $\bar{p}^{(S T)}=250 \mathrm{MW}$, similar to the Siemens SST-3000 turbine [46]. We assume that the turbine can be operated at full power over the entire planning horizon, i.e. the state constraints (19) are omitted. In contrast to the freezer discussed in Section VI-B, the turbine is assumed to be running at an operating point where it can react to power set point changes without delays, i.e., $\delta^{(S T)}=0$. However, the rate at which the turbine power can be varied is limited by the power ramp-rate constraints $-\underline{r}^{(S T)}=\bar{r}^{(S T)}=4.5 \mathrm{MW} / \mathrm{min}$ [11]. These ramprate limits determine the maximum SFR capacity the turbine can offer. The accurate tracking of the target power trajectory (4) requires that, in the extreme case, the power output of the turbine must be changed by $\pm 2 \gamma^{(S T)}$ over the course of one SFR time step $T^{\mathrm{RES}}=10 \mathrm{~s}$. These ramping requirements must not exceed the turbine's power ramp-rate limits, i.e.,

$$
2 \gamma_{\max }^{(S T)} / T^{\mathrm{RES}} \leq \min \left\{\bar{r}^{(S T)},-\underline{r}^{(S T)}\right\} .
$$

The constraint (29) determines the maximum SFR capacity the steam turbine can provide, namely, $\gamma_{\max }^{(S T)}=375 \mathrm{~kW}$, which corresponds to $0.15 \%$ of $\bar{p}^{(S T)}$.

We compute the maximum SFR capacity available from an aggregation of batteries and a steam turbine by solving the bidding problem (24) without the control delay constraints (24g) for different choices of battery parameters $\left(\bar{p}^{(B)}, \bar{x}^{(B)}\right)$. The resulting aggregate SFR capacities and corresponding synergy factors are provided in Table III. The aggregation of the batteries and the steam turbine yield synergy factors in the range of $0.18-1.25$, i.e., it can provide $18-125 \%$ more SFR capacity than the batteries and the turbine could provide individually. The two types of systems complement each other: if needed, the turbine can exchange energy with the batteries via adjustments of the reference schedules (7) to keep the state-of-charge of the batteries feasible. The batteries, on the other hand, take over the high-frequency component of the SFR activation signal and thereby reduce the thermal stress and operations and maintenance costs of the turbine that can results from frequent ramp-ups and ramp-downs.

TABLE III

BATTERY-STEAM TURBine AgGREgAtion

\begin{tabular}{lcccccc}
\hline Battery type & $\#$ & $\begin{array}{c}\bar{p}^{(B)} \\
\mathrm{kW}\end{array}$ & $\begin{array}{c}\bar{x}^{(B)} \\
\mathrm{MWh}\end{array}$ & $\begin{array}{c}\gamma_{\max }^{(\mathrm{B})} \\
\mathrm{kW}\end{array}$ & $\begin{array}{c}\gamma_{\max }^{(\mathrm{agg})} \\
\mathrm{kW}\end{array}$ & $\sigma^{\text {SFR }}$ \\
\hline \hline Model-S & 10 & 172 & 1 & 20.83 & 468.70 & 0.18 \\
& 50 & 860 & 5 & 104.17 & 843.50 & 0.76 \\
& 100 & 1720 & 10 & 208.33 & 1312.00 & 1.25 \\
\hline Powerpack & 5 & 250 & 1.05 & 21.88 & 506.84 & 0.28 \\
& 10 & 500 & 2.1 & 43.75 & 638.68 & 0.53 \\
& 20 & 1000 & 4.2 & 87.50 & 902.35 & 0.95 \\
\hline Powerwall & 50 & 350 & 0.675 & 14.06 & 551.00 & 0.42 \\
& 100 & 700 & 1.35 & 28.13 & 726.99 & 0.80 \\
\hline
\end{tabular}




\section{CONCLUSION}

This work considered the energy and reserve bidding problem of an AG trading energy and offering SFR services on behalf of a group of flexible systems. The problem was formulated as a robust optimization problem whose solution comprises optimal affine control policies that the AG can apply directly. The problem formulation explicitly takes into account power ramp-rate limits and control delays of individual systems, as well as all the different timescales involved in the bidding problem. This makes the approach versatile and applicable to various market settings. Simulation results demonstrate that aggregating flexible systems with complementary physical properties can free up additional SFR capacity in the range of $18-1190 \%$ compared with the amount of reserves the systems could provide individually. This synergy effect can unlock the regulation potential of different types of energy resources with control delays or restrictive energy or ramp-rate constraints.

There are several future directions of interest. First, the energy and reserve scheduling problem can be augmented to consider additional types of ancillary services, such as spinning reserves and ramping capacity services. In the bidding problem, the AG must consider all the different timescales of the services and the corresponding markets and ensure the feasibility of all individual target power trajectories even if multiple services are activated simultaneously. Second, the AG is currently assumed to be a price taker. Future work could investigate how to find optimal price-capacity bids and evaluate the synergy effect with regard to the AG's economic profit. The pricing of SFR capacity could be based on flexibility cost functions that reflect the costs of individual systems deviating from their reference schedules. Third, the requirement to track SFR activation signals exactly can be relaxed to track the signals only as accurately as required by the SO, for example with regard to regulation mileage constraints or other performance-based measures [31], [35]. Further, the incorporation of distribution network constraints [47], [48] into the bidding problem should be studied.

\section{ACKNOWLEDGMENT}

The authors gratefully acknowledge the fruitful discussions with Bernhard Jansen.

\section{REFERENCES}

[1] Swissgrid, "Basic principles of ancillary service products," Swissgrid, Tech. Rep., 2017. [Online]. Available: https://www.swissgrid.ch/dam/swissgrid/experts/ancillary_ services/Dokumente/D170214_AS-Products_V9R2_en.pdf

[2] ENTSO-E, Load-frequency control and performance. ENTSOE, 2004. [Online]. Available: https://www.entsoe.eu/resources/ publica-tions/system-operations/operation-handbook/

[3] E. Vrettos, F. Oldewurtel, F. Zhu, and G. Andersson, "Robust provision of frequency reserves by office building aggregations," in 19th World Congr. Int. Fed. Autom. Control, vol. 19. IFAC, 2014, pp. 12068 12073.

[4] F. A. Qureshi, I. Lymperopoulos, A. Ahmadi Khatir, and C. N. Jones, "Economic advantages of office buildings providing ancillary services with intraday participation," IEEE Trans. Smart Grid, no. 99, pp. 1-10, 2016.
[5] H. Hao, A. Kowli, Yashen Lin, P. Barooah, and S. Meyn, "Ancillary service for the grid via control of commercial building HVAC systems," in Americ. Control Conf. IEEE, 2013, pp. 467-472.

[6] Y. Lin, P. Barooah, and S. Meyn, "Low-frequency power-grid ancillary services from commercial building HVAC systems," in IEEE Int. Conf. Smart Grid Comm. IEEE, 2013, pp. 169-174.

[7] S. I. Vagropoulos and A. G. Bakirtzis, "Optimal bidding strategy for electric vehicle aggregators in electricity markets," IEEE Trans. Power Syst., vol. 28, no. 4, pp. 4031-4041, 2013.

[8] H. Liu, Z. Hu, Y. Song, J. Wang, and X. Xie, "Vehicle-to-grid control for supplementary frequency regulation considering charging demands," IEEE Trans. Power Syst., vol. 30, no. 6, pp. 3110-3119, 2014.

[9] H. Hao, B. M. Sanandaji, K. Poolla, and T. L. Vincent, "A generalized battery model of a collection of thermostatically controlled loads for providing ancillary service," in 51st Allert. Conf. Commun., Control Comput. IEEE, 2013, pp. 551-558.

[10] T. Borsche, A. Ulbig, M. Koller, and G. Andersson, "Power and energy capacity requirements of storages providing frequency control reserves," in IEEE PES Gen. Meet. IEEE, 2013, pp. 1-5.

[11] Y. V. Makarov, S. Lu, J. Ma, and T. B. Nguyen, "Assessing the value of regulation resources based on their time response characteristics," Pacific Northwest National Laboratory, Tech. Rep., 2008. [Online]. Available: https://www.pnnl.gov/main/publications/ external/technical_reports/PNNL-17632.pdf

[12] H. Nosair and F. Bouffard, "Energy-centric flexibility management in power systems," IEEE Trans. Power Syst., vol. 31, no. 6, pp. 50715081, 2016.

[13] I. Lymperopoulos, F. A. Qureshi, T. Nghiem, A. A. Khatir, and C. N. Jones, "Providing ancillary service with commercial buildings: The Swiss perspective," in Int. Fed. Autom. Control, vol. 48, no. 8. Elsevier, 2015, pp. 6-13.

[14] E. Vrettos, F. Oldewurtel, and G. Andersson, "Robust energy-constrained frequency reserves from aggregations of commercial buildings," IEEE Trans. Power Syst., vol. 31, no. 6, pp. 4272-4285, 2016.

[15] T. Borsche, A. Ulbig, and G. Andersson, "A new frequency control reserve framework based on energy-constrained units," in Power Syst. Comput. Conf. IEEE, 2014, pp. 1-7.

[16] L. Fabietti, T. T. Gorecki, F. A. Qureshi, A. Bitlislioglu, I. Lymperopoulos, and C. N. Jones, "Experimental implementation of frequency regulation services using commercial buildings," IEEE Trans. Smart Grid, pp. 1-9, 2016.

[17] F. Rey, X. Zhang, S. Merkli, V. Agliati, M. Kamgarpour, and J. Lygeros, "Strengthening the group: Aggregated frequency reserve bidding with ADMM," IEEE Trans. Smart Grid, pp. 1-10, 2018.

[18] L. Gkatzikis, I. Koutsopoulos, and T. Salonidis, "The role of aggregators in smart grid demand response markets," IEEE J. Sel. Areas Commun., vol. 31, no. 7, pp. 1247-1257, 2013.

[19] S. Koch and G. Andersson, "Assessment of revenue potentials of ancillary service provision by flexible unit portfolios," in IEEE PES Gen. Meet. IEEE, 2012, pp. 1-8.

[20] X. Zhang, E. Vrettos, M. Kamgarpour, G. Andersson, and J. Lygeros, "Stochastic frequency reserve provision by chance-constrained control of commercial buildings," in Eur. Control Conf., 2015, pp. 1134-1140.

[21] J. Warrington, P. Goulart, S. Mariéthoz, and M. Morari, "Policy-based reserves for power systems," IEEE Trans. Power Syst., vol. 28, no. 4, pp. 4427-4437, 2013.

[22] T. T. Gorecki, L. Fabietti, F. A. Qureshi, and C. N. Jones, "Experimental demonstration of buildings providing frequency regulation services in the Swiss market," Energy Build., vol. 144, pp. 229-240, 2017.

[23] J. Warrington, P. J. Goulart, S. Mariethoz, and M. Morari, "Robust reserve operation in power systems using affine policies," in 51st IEEE Conf. Decis. Control. IEEE, 2012, pp. 1111-1117.

[24] D. Bertsimas, E. Litvinov, X. A. Sun, J. Zhao, and T. Zheng, "Adaptive robust optimization for the security constrained unit commitment problem," IEEE Trans. Power Syst., vol. 28, no. 1, pp. 52-63, 2013.

[25] A. Ben-Tal, A. Goryashko, E. Guslitzer, and A. Nemirovski, "Adjustable robust solutions of uncertain linear programs," Math. Program., vol. 99, no. 2, pp. 351-376, 2004.

[26] G. Morales-Espana, A. Ramos, and J. Garcia-Gonzalez, "An MIP formulation for joint market-clearing of energy and reserves based on ramp scheduling," IEEE Trans. Power Syst., vol. 29, no. 1, pp. 476-488, 2014.

[27] M. Parvania and A. Scaglione, "Unit commitment with continuous-time generation and ramping trajectory models," IEEE Trans. Power Syst., vol. 31, no. 4, pp. 3169-3178, 2016. 
[28] FERC, "Frequency regulation compensation in the organized wholesale power markets," U.S. Federal Energy Regulatory Commission, Tech. Rep., 2011. [Online]. Available: https://www.ferc.gov/whats-new/ comm-meet/2011/102011/e-28.pdf

[29] L. Zhang, F. Jabbari, T. Brown, and S. Samuelsen, "Coordinating plugin electric vehicle charging with electric grid: Valley filling and target load following," J. Power Sources, vol. 267, pp. 584-597, 2014.

[30] F. L. Müller, S. Woerner, and J. Lygeros, "Ramp-rate-constrained bidding of energy and frequency reserves in real market settings," pp. 1-8, 2018. [Online]. Available: https://arxiv.org/abs/1804.03892

[31] A. Sadeghi-Mobarakeh and H. Mohsenian-Rad, "Performance accuracy scores in CAISO and MISO regulation markets: A comparison based on real data and mathematical analysis," IEEE Trans. Power Syst., vol. 33 , no. 3, pp. 3196-3198, 2018.

[32] T. Wei, B. Zheng, Q. Zhu, and S. Hu, "Proactive demand participation of smart buildings in smart grid," IEEE Trans. Comput., vol. 65, no. 5, pp. 465-472, 2016.

[33] M. Gonzalez Vaya and G. Andersson, "Optimal bidding strategy of a plug-in electric vehicle aggregator in day-ahead electricity markets," IEEE Trans. Power Syst., vol. 30, no. 5, pp. 2375-2385, 2015.

[34] D. Bertsimas, D. B. Brown, and C. Caramanis, "Theory and applications of robust optimization," Soc. Ind. Appl. Math., vol. 53, no. 3, pp. 464 501, 2011.

[35] C. Hinman, "Pay for performance regulation (FERC Order 755): Updated with year one design changes," CAISO, Tech. Rep., 2015. [Online]. Available: https://www.caiso.com/Documents/ Pay-PerformanceRegulationFERC_Order755Presentation.pdf

[36] D. Schlipf, M. Scherer, and M. Haller, "Test for secondary control capability," Swissgrid, Tech. Rep., 2012. [Online]. Available: https://www.swissgrid.ch/dam/swissgrid/experts/ancillary_services/ prequalification/en/D130422_Test-for-secon-dary-control-capability_ V2R1_EN.pdf

[37] A. Bitlislioglu, T. T. Gorecki, and C. N. Jones, "Robust tracking commitment," IEEE Trans. Autom. Control, vol. 62, no. 9, pp. 44514466, 2017.

[38] B. Foggo and N. Yu, "Improved battery storage valuation through degradation reduction," IEEE Trans. Smart Grid, vol. 3053, pp. 1-13, 2017.

[39] N. Kumar, B. Peter, S. Lefton, D. Agan, and D. Hilleman, "Power plant cycling costs," NREL, Tech. Rep., 2012. [Online]. Available: https://www.nrel.gov/docs/fy12osti/55433.pdf

[40] M. Balandat, F. Oldewurtel, M. Chen, and C. Tomlin, "Contract design for frequency regulation by aggregations of commercial buildings," in 52nd Allert. Conf. Comm., Control. Comput. IEEE, 2014, pp. 38-45.

[41] F. L. Müller, O. Sundström, J. Szabo, and J. Lygeros, "Aggregation and disaggregation of energetic flexibility from distributed energy resources," IEEE Trans. Smart Grid, pp. 1-10, 2017.

[42] "Tesla Model S," 2018. [Online]. Available: https://www.tesla.com/ models

[43] F. L. Müller, O. Sundström, C. Binding, and D. Dykeman, "Power reference tracking of a large-scale industrial freezer system for ancillary service delivery," in IEEE PES Innov. Smart Grid Technol. Eur. IEEE, 2013, pp. 1-5.

[44] “Tesla Powerpack," 2018. [Online]. Available: https://www.tesla.com/ powerpack

[45] Tesla, "Power Wall 2 AC," 2016. [Online]. Available: http://www.energymatters.com.au/wp-content/uploads/2016/11/ tesla-powerwall-2-datasheet.pdf

[46] Siemens, "Siemens steam turbine portfolio," Siemens, Tech. Rep., 2017. [Online]. Available: https://www.energy.siemens. $\mathrm{com} / \mathrm{mx} /$ pool/hq/power-generation/steam-turbines/downloads/new/ siemens-steam-turbines-product-overview-EN-updated.pdf

[47] W. Wang and N. Yu, "Chordal conversion based convex iteration algorithm for three-phase optimal power flow problems," IEEE Trans. Power Syst., vol. 33, no. 2, pp. 1603-1613, 2018.

[48] E. Dall'Anese, Hao Zhu, and G. B. Giannakis, "Distributed optimal power flow for smart microgrids," IEEE Trans. Smart Grid, vol. 4, no. 3 , pp. 1464-1475, 2013.
Fabian Müller received the B.Sc. degree in mechanical engineering and the M.Sc. degree in robotics, systems and control from ETH Zurich, Switzerland, in 2009 and 2012, respectively.

He was recently affiliated with the Institute for Dynamic Systems and Control at ETH Zurich.

Mr. Müller is currently a doctoral student with the Automatic Control Laboratory at ETH Zurich and with IBM Research-Zurich. His research interests include smart power grids, control theory, and optimization.

Stefan Woerner received the M.Sc degree in applied mathematics and the doctoral degree from the Department of Management, Technology and Economics, ETH Zurich, Switzerland, in 2010 and 2013, respectively.

$\mathrm{He}$ is currently a research staff member at IBM Research-Zurich where he leads multiple projects on supply chain management and forecasting.

Dr. Woerner's main research interests are in approximate dynamic programming, robust optimization, simulation optimization, and applications of these techniques to problems in supply chain management and energy systems.

John Lygeros ( $\left.F^{\prime} 11\right)$ received the B.Eng. degree in electrical engineering and the M.Sc. degree in systems control from the Imperial College of Science Technology and Medicine, London, U.K., in 1990 and 1991, respectively, and the Ph.D. degree from the Electrical Engineering and Computer Sciences Department, University of California at Berkeley (UC Berkeley), Berkeley, CA, USA, in 1996

After Post-Doctoral Researcher appointments at MIT and UC Berkeley in 2000, he joined the Department of Engineering, University of Cambridge, Cambridge, U.K., as a Lecturer and Churchill College, Cambridge, as a fellow. From 2003 to 2006, he was an Assistant Professor with the Department of Electrical and Computer Engineering, University of Patras, Patras, Greece. In 2006, he joined the Automatic Control Laboratory, ETH Zurich, Zürich, Switzerland, as an Associate Professor, where he has been a Full Professor since 2010. Since 2009, he has been serving as the Head of the Automatic Control Laboratory, and since 2015, he has been serving as the Head of the Department of Information Technology and Electrical Engineering. His current research interests include modeling, analysis, and control of hierarchical, hybrid, and stochastic systems, with applications to biochemical networks, automated highway systems, air traffic management, power grids, and camera networks.

Dr. Lygeros is a member of the IET and the Technical Chamber of Greece. Since 2013, he has been serving as the Treasurer of the International Federation of Automatic Control. 